\title{
Codes on Algebraic Curves
}




\section{Codes on Algebraic Curves}

Serguei A. Stepanov

Bilkent University

Ankara, Turkey

and Steklov Mathematical Institute

Moscow, Russia

Springer Science+Business Media, LLC 


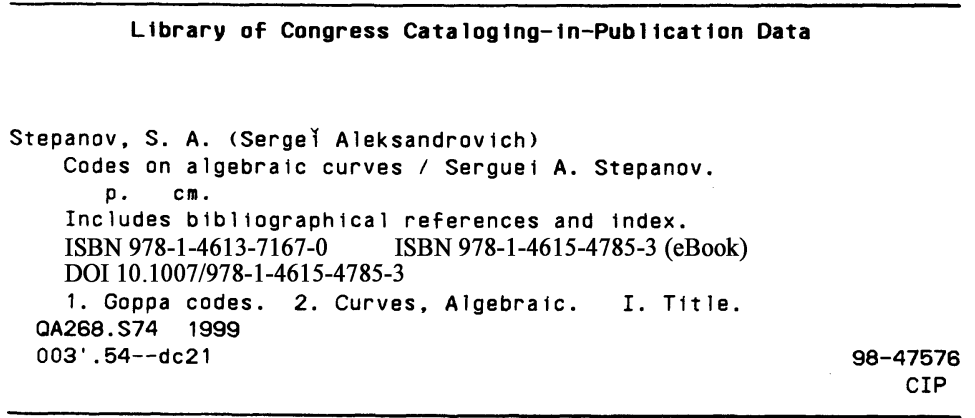

ISBN 978-1-4613-7167-0

(C) 1999 Springer Science+Business Media New York

Originally published by Kluwer Academic/Plenum Publishers in 1999

Softcover reprint of the hardcover 1 st edition 1999

10987654321

A C.I.P. record for this book is available from the Library of Congress.

All rights reserved

No part of this book may be reproduced, stored in a retrieval system, or transmitted in any form or by any means, electronic, mechanical, photocopying, microfilming, recording, or otherwise, without written permission from the Publisher 


\section{Preface}

This is a self-contained introduction to algebraic curves over finite fields and geometric Goppa codes. There are four main divisions in the book. The first is a brief exposition of basic concepts and facts of the theory of error-correcting codes (Part I). The second is a complete presentation of the theory of algebraic curves, especially the curves defined over finite fields (Part II). The third is a detailed description of the theory of classical modular curves and their reduction modulo a prime number (Part III). The fourth (and basic) is the construction of geometric Goppa codes and the production of asymptotically good linear codes coming from algebraic curves over finite fields (Part IV).

The theory of geometric Goppa codes is a fascinating topic where two extremes meet: the highly abstract and deep theory of algebraic (specifically modular) curves over finite fields and the very concrete problems in the engineering of information transmission. At the present time there are two essentially different ways to produce asymptotically good codes coming from algebraic curves over a finite field with an extremely large number of rational points. The first way, developed by M. A. Tsfasman, S. G. Vladut and Th. Zink [210], is rather difficult and assumes a serious acquaintance with the theory of modular curves and their reduction modulo a prime number. The second way, proposed recently by A. Garcia and H. Stichtenoth $[53,54,56]$, is much easier and more explicit; the basic tools are the ramification theory of Artin-Schreier extensions and the Hurwitz genus formula. This book demonstrates both of these ways. Moreover, it contains various examples of particular geometric Goppa codes of admissible length, which have fairly good parameters and can be easily used in practice. For example, the author's recent constructions [188,189] of linear codes on fiber products of hyperelliptic curves provides a family of sufficiently long codes with completely 
good parameters and easy construction and decoding algorithms.

Recently, a series of effective decoding algorithms for geometric Goppa codes was worked out by several authors. Such algorithms decode up to half the minimum distance and have polynomial complexity. This book provides a detailed description of the most significant results on the decoding of geometric Goppa codes and concrete realizations of various decoding algorithms in the simplest case of plane projective curves.

My purpose is to present these themes in a simple, easily understandable manner, and also to explain their close interconnection. At the same time I want to introduce topics which are at the forefront of current research. Numerous examples are given in the text and exercises, with the aim of making the material readable and interesting to mathematicians in fields far removed from the subject of the book. Some exercises are rather difficult and are intended for actively working readers.

This book grew out of lectures I gave at the Institute of Mathematics of Academia Sinica (Beijing) in January-April of 1992. The excellent book of J. H. van Lint and G. B. M. van der Geer, Introduction to Coding Theory and Algebraic Geometry [116], served as a guideline for organizing the material. Some constructions were adopted from a fundamental (but rather difficult for nonspecialists in algebraic geometry) work by M. A. Tsfasman and S. G. Vladut, Algebraic-Geometric Codes [208] and from the author's monograph Arithmetic of Algebraic Curves [187]. The presentation of the theory of classical modular curves and the construction of asymptotically good codes coming from these curves are fairly close to the approaches in N. Koblitz's, Introduction to Elliptic Curves and Modular Forms [96] and C. Moreno's, Algebraic Curves over Finite Fields [129]. The excellent survey article of Hoholdt and Pellikaan, On the Decoding of Algebraic-Geometric Codes [80], was extensively consulted to describe the contemporary state of the decoding of geometric Goppa codes. The book also contains a brief exposition of the theory of algebraic function fields over a finite constant field (in particular, the Artin-Schreier extensions of the rational function field). For a more detailed treatment of this theory see M. Deuring's, Lectures on the Theory of Algebraic Functions of One Variable [24] and H. Stichtenoth's, Algebraic Function Fields and Codes [197].

In order to be able to read this book a fairly thorough mathematical background is necessary. The most important area is certainly algebra (especially linear algebra and Galois theory), but the reader must also know some facts from elementary number theory, complex analysis and the theory of finite fields. For these I refer the reader to standard textbooks and also to R. Lidl and H. Niederreiter's, Finite Fields [114]. For a more extensive treatment of coding theory and the theory of modular curves I strongly recommend: F. J. MacWilliams and N. J. A. Sloane's, The Theory of Error-Correcting Codes [118], and G. Shimura's, Introduction to the Arithmetic Theory of Automorphic Functions [176]. 
I would like to express my gratitude to everyone at the Institute of Mathematics of Academia Sinica for their hospitality. I would especially like to thank Wang Yuan, Yang Lo and Feng Xu-ning for their constant encouragement and help during my stay in Beijing.

I wish to express my gratitude to many people at Bilkent University (Ankara) for invaluable help in the final stage of preparation of the manuscript. Specifically, I would like to thank Alexander Klyachko, Vladimir Kurakin and Sinan Sertöz for their careful reading of the original draft and their many useful comments. Finally, I wish to thank Ferruh Özbudak for having typed most of the chapters and Theresa Caner for having proofread. 


\section{Contents}

\section{Error-Correcting Codes}

Chapter 1

Codes and Their Parameters 3

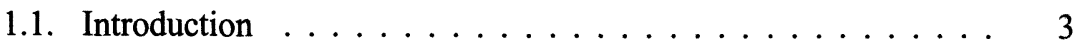

1.2. Finite Fields $\ldots \ldots \ldots \ldots \ldots \ldots \ldots \ldots \ldots \ldots \ldots$

1.3. Linear Codes . . . . . . . . . . . . . . . . . 13

1.4. Spectrum and Duality . . . . . . . . . . . . . 15

Exercises ...................... 20

Chapter 2

Bounds on Codes

2.1. Upper Bounds . . . . . . . . . . . . . . . . 26

2.2. The Linear Programming Bound $\ldots \ldots \ldots \ldots \ldots \ldots$

2.3. Lower Bounds . . . . . . . . . . . . . . . . . 35

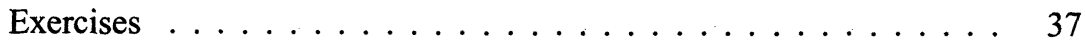

Chapter 3

Examples and Constructions 41

3.1. Codes of Genus Zero . . . . . . . . . . . . . . . . . . . . 41

3.2. Some Families of Codes . . . . . . . . . . . . . . 46

3.3. Constructing Codes from other Codes . . . . . . . . 60

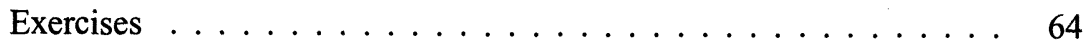




\section{Algebraic Curves and Varieties}

Chapter 4

Algebraic Curves $\quad 71$

4.1. Algebraic Varieties ................... 71

4.2. Non-Singular Curves ... . . . . . . . . . . . . . . 77

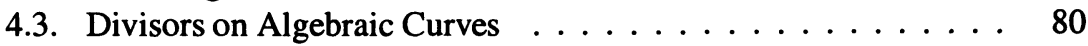

4.4. The Riemann-Roch Theorem . . . . . . . . . . . . 85

4.5. Hurwitz and Plücker Genus Formulas . . . . . . . . . . . . . . . . . 93

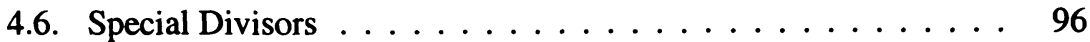

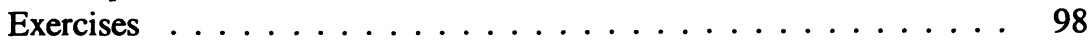

Chapter 5

Curves over a Finite Field 103

5.1. Rational Points and Divisors . . . . . . . . . . . . . . . 105

5.2. The Zeta-Function of a Curve .............. . . 111

5.3. L-Functions of Artin . . . . . . . . . . . . . . . . . . 120

5.4. Algebraic Function Fields . . . . . . . . . . . . . . . . . . . . . 130

Exercises ........................... 139

Chapter 6

Counting Points on Curves over Finite Fields 143

6.1. The Number of Rational Points on a Curve . . . . . . . . . . . 143

6.2. Character Sums . . . . . . . . . . . . . . . . . . . . 147

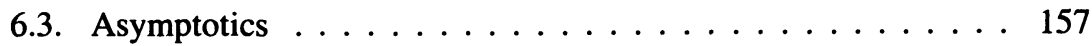

Exercises ........................ 170

\section{Elliptic and Modular Curves}

\section{Chapter 7}

Elliptic Curves $\quad 175$

7.1. The Group Law . . . . . . . . . . . . . . . . . . . . . . . . . . . . . . . . . 175

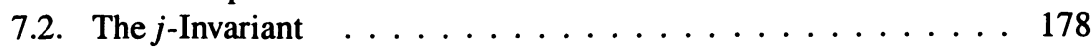

7.3. Isogenies . . . . . . . . . . . . . . . . . . 180

7.4. Elliptic Curves over Finite Fields . . . . . . . . . . . . . . 184

7.5. Elliptic Functions . . . . . . . . . . . . . . . . 186

Exercises .......................... 190

\section{Chapter 8}

$\begin{array}{lc}\text { Classical Modular Curves } & 193\end{array}$

8.1. Congruence Subgroups . . . . . . . . . . . . . . . . . 193

8.2. The Curves $X(N), X_{0}(N)$, and $X_{1}(N) \ldots \ldots \ldots$ 
8.3. Hecke Operators . . . . . . . . . . . . . . . . . . . . . 199

8.4. The Petersson Inner Product . . . . . . . . . . . . . . . 212

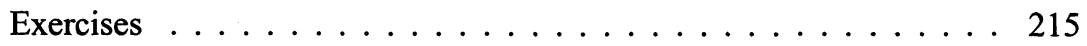

Chapter 9

Reductions of Modular Curves $\quad 219$

9.1. Reductions and Moduli Spaces . . . . . . . . . . . . 219

9.2. The Igusa Theorem . . . . . . . . . . . . . . . . . . . 224

9.3. The Eichler-Shimura Congruence Relation . . . . . . . . 231

9.4. The Eichler-Selberg Trace Formula . . . . . . . . . . . . 236

Exercises . . . . . . . . . . . . . . . . . . . 239

\section{Geometric Goppa Codes}

Chapter 10

Constructions and Properties 243

10.1. L-Construction . . . . . . . . . . . . . . . . 243

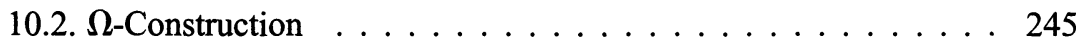

10.3. Parameters . . . . . . . . . . . . . . . . 248

10.4. Duality and Spectra . . . . . . . . . . . . . . 251

Exercises . . . . . . . . . . . . . . . . . 254

Chapter 11

Examples 257

11.1. Codes of Small Genera . . . . . . . . . . . . . . . . . 257

11.2. Elliptic and Hermitian Codes _. . . . . . . . . . . 261

11.3. Codes on Fiber Products . . . . . . . . . . . . . . . . . 267

11.4. Codes on Classical Modular Curves . . . . . . . . . . . . . 274

11.5. Codes on Artin-Schreier Coverings . . . . . . . . . . . . 276

11.6. Codes on Trace-Norm Curves . . . . . . . . . . . . . . . . 284

Exercises . . . . . . . . . . . . . . . . . . . . 287

\section{Chapter 12}

Decoding Geometric Goppa Codes 289

12.1. The Decoding Problem . . . . . . . . . . . . . . . . . . . 289

12.2. The Basic and Modified Algorithms . . . . . . . . . . 292

12.3. An Improvement of the Modified Algorithm . . . . . . . . . . . 301

12.4. Majority Voting for Unknown Syndromes . . . . . . . . . . . . 306

12.5. Faster Decoding . . . . . . . . . . . . . . . . . . . 309

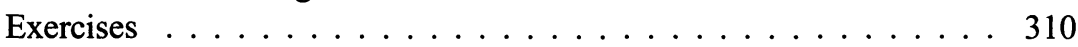


Chapter 13

Bounds

13.1. Asymptotic Bounds . . . . . . . . . . . . . . . . 315

13.2. Constructive Bounds . . . . . . . . . . . . 316

13.3. Other Bounds . . . . . . . . . . . . . . . . . 319

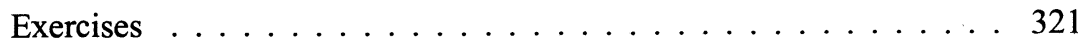

Bibliography 323

$\begin{array}{ll}\text { List of Notations } & 335\end{array}$

Index 343 


\section{Codes on Algebraic Curves}

